\title{
Identification of diverse activating mutations of the RAS-MAPK pathway in histiocytic sarcoma
}

\author{
Vignesh Shanmugam ${ }^{1,2} \cdot$ Gabriel K. Griffin $^{1,2} \cdot$ Eric D. Jacobsen ${ }^{2,3} \cdot$ Christopher D. M. Fletcher $^{1,2} \cdot$ Lynette M. Sholl $^{1,2}$ • \\ Jason L. Hornick $\mathbb{B}^{1,2}$
}

Received: 22 October 2018 / Revised: 24 November 2018 / Accepted: 24 November 2018 / Published online: 9 January 2019

(c) United States \& Canadian Academy of Pathology 2019

\begin{abstract}
Recent studies have demonstrated recurrent activating mutations involving the classical MAPK and PI3K signaling pathways in a large proportion of histiocytic neoplasms, such as Langerhans cell histiocytosis. However, very little is known about the molecular genetics of histiocytic sarcoma, a rare aggressive malignant neoplasm that shows pathologic characteristics of mature macrophages. Here we report the genomic characteristics of a large cohort of histiocytic sarcomas $(n=28)$ using a targeted next-generation sequencing approach to identify driver alterations. We identified recurrent mutations involving the RAS-MAPK signaling pathway (MAP2K1, KRAS, NRAS, BRAF, PTPN11,NF1, CBL) in a majority (57\%) of histiocytic sarcoma cases and report a clinical response to a MEK inhibitor (Cobimetinib) in a patient with a NF1mutated histiocytic sarcoma. A smaller subset of cases $(21 \%)$ also showed mutations resulting in activation of the PI3K signaling pathway (PTEN, MTOR, PIK3R1, PIK3CA). In addition, the tumor-suppressor gene CDKN2A was the most frequently altered gene $(46 \%)$. Further, a subset of histiocytic sarcoma cases shows striking molecular genetic similarities to B cell lymphomas, supporting a clonal relationship between B cell neoplasms and a subset of histiocytic sarcomas. These findings support a cooperative role for MAPK, PI3K, and cyclin-CDK4/6-INK4 signaling in the pathogenesis of histiocytic sarcoma and provide a rational basis for targeting these pathways.
\end{abstract}

\section{Introduction}

The histiocytic/dendritic cell neoplasms are a heterogeneous group of tumors characterized by tissue infiltration by neoplastic cells of macrophage/dendritic cell lineage $[1,2]$. Recent studies have demonstrated recurrent activating mutations involving the classical mitogen-activated protein kinase (MAPK) and phosphoinositide-3 kinase (PI3K)/

Co-senior authors: Lynette M. Sholl, Jason L. Hornick

Supplementary information The online version of this article (https:// doi.org/10.1038/s41379-018-0200-x) contains supplementary material, which is available to authorized users.

Jason L. Hornick

jhornick@bwh.harvard.edu

1 Department of Pathology, Brigham and Women's Hospital, Boston, MA, USA

2 Harvard Medical School, Boston, MA, USA

3 Center for Hematologic Oncology, Dana Farber Cancer Institute, Boston, MA, USA
AKT/mammalian target of rapamycin (MTOR) signaling pathways in Langerhans cell histiocytosis and Erdheim-Chester disease, two of the most common of the histiocytic/dendritic cell neoplasms. The identification of recurrent BRAFV600E mutations in these tumors led to the study of vemurafenib (RAF kinase inhibitor) for the treatment of BRAFV600E-mutated histiocytoses [3, 4] and its eventual approval for the treatment of Erdheim-Chester disease by the Food and Drug Administration.

However, very little is known about the molecular genetics of histiocytic sarcoma, one of the most aggressive histiocytic neoplasms. Histiocytic sarcoma is an extremely rare malignant neoplasm of presumed myeloid origin that shows histopathologic characteristics of mature macrophages/ histiocytes [5]. A subset of these neoplasms can arise through clonal evolution of pre-existing hematologic neoplasms [6, 7]. There is no standardized therapy for histiocytic sarcoma. Despite the lack of prospective clinical data, combination chemotherapy regimens similar to those used in the treatment of non-Hodgkin lymphomas are often used. However, response rates to these regimens are mixed with frequent resistance to therapy [8]. The lack of a comprehensive 
understanding of the molecular genetics of histiocytic sarcoma has hindered the use of rational targeted therapies.

The goal of this study was to systematically evaluate the genomic characteristics of histiocytic sarcoma using a targeted next-generation sequencing approach to better understand the biology of this rare aggressive tumor and also to uncover potential targets for therapy. Here we report the genomic characterization of a large cohort of histiocytic sarcomas and the findings from an index patient who experienced a clinical response after receiving genomically directed targeted therapy.

\section{Methods}

\section{Patient identification and tumor samples}

After appropriate institutional review board approval, all cases of histiocytic sarcoma diagnosed at Brigham and Women's Hospital over an 18-year period (2000-2018) were identified. Patients who received preoperative chemotherapy or radiotherapy were excluded. All slides were retrospectively reviewed and diagnoses were independently confirmed by two experienced pathologists. Only cases that showed prominent atypia and mitotic activity with strong expression of at least two macrophage lineage markers (CD163, CD68, lysozyme, PU.1/SPI1, CD4) were included. Cases with at least $20 \%$ tumor cellularity were included to yield sufficient DNA for sequencing. Microdissection was necessary in a subset of cases to enrich for tumor DNA.

\section{Targeted next-generation sequencing}

Targeted sequencing of all exonic and selected intronic regions of 447 cancer-associated genes (OncoPanel POPv3) was performed on nearly all cases (25/28) using hybrid capture with a custom probe set (Agilent SureSelect, Agilent Technologies, Santa Clara, CA) and massively parallel sequencing (HiSeq 2500, Illumina, San Diego, CA), as previously described [9]. For three cases, sequencing data were obtained from an older version of our next-generation sequencing panel (OncoPanel POPv2). Most of the genes covered by POPv2 were also covered by POPv3 (200/250; see supplementary tables for full list).

\section{Data processing and variant classification}

Data processing and analysis were performed using MuTect and GATK for single-nucleotide variants [10, 11], Indelocator for insertions and deletions (http://archive.broadinstitute.org/ca ncer/cga/indelocator), an internally developed algorithm (RobustCNV) for copy-number variations (Dana Farber Cancer Institute, Boston, MA), and BreakMer for large structural variations [12]. The validated analytical sensitivity for the detection of single-nucleotide variants is $~ 10 \%$ variant allele fraction. Manual review and annotation were performed for all single-nucleotide variants, copy-number variations, and translocation calls made by the bioinformatics pipeline. Variants that were previously reported as single-nucleotide polymorphisms in gnomAD were excluded as likely germline variants. Variants were classified as pathogenic if one or more of the following conditions were met: (1) reported in COSMIC as a recurrent event, (2) reported in OncoKB as at least likely oncogenic, (3) compelling evidence of biological effect in the literature. The remaining variants were classified as variants of uncertain significance. The following copy-number alterations were classified as pathogenic: amplifications, homozygous deletions, concurrent copy-number gain/loss and pathogenic mutation involving the same gene (e.g., loss of heterozygosity, amplification of allele bearing an activating mutation) and focal (1-5 genes) copy gains or losses of oncogenes/tumor-suppressor genes that are shared across the cohort ( $>10 \%$ of cases). The variant allele fractions were normalized for variability in tumor purity ("corrected" variant allele fraction) by dividing the allele fraction by the tumor purity (by morphologic/immunohistochemical assessment).

\section{Aberrant somatic hypermutation analysis}

To investigate for evidence of aberrant somatic hypermutation, we manually reviewed all variants that were identified in a set of previously reported target genes: BCL6, BCL2, CIITA, PAX5, MYC, PIM1, SOCS1 (aberrant somatic hypermutation target genes) [13]. We then excluded all variants with an allele frequency of $\geq 40 \%$ to exclude germline single-nucleotide polymorphisms. Additionally, the number of mutations involving WRCY/RGYW ( $\mathrm{W}=\mathrm{A}$ or $\mathrm{T}, \mathrm{R}=\mathrm{A}$ or $\mathrm{G}, \mathrm{Y}=\mathrm{C}$ or $\mathrm{T})$ motifs were also quantified for each case. Mutation density at these target genes was calculated by dividing the total number of variants identified in these genes by the total number of base pairs of target genes covered. Cases with mutation density greater than the third quartile were classified as showing an "aberrant somatic hypermutation signature." The mutations identified in these cases were further characterized to demonstrate that they showed features of those in B cell lymphomas with evidence of aberrant somatic hypermutation, i.e., recurrent single-nucleotide substitutions involving a set of target genes, located within $2 \mathrm{~kb}$ distal to the transcription start site, bias for transition over transversion mutations, and WRCY/RGYW motif bias [13].

The WRCY enrichment/motif bias ratio was calculated by dividing the observed number of mutations by the expected number of mutations at WRCY motifs. The expected number of mutations in a WRCY motif is calculated by taking the percentage of bases occurring at WRCY motifs in a given target region and multiplying it by the total 
number of single-nucleotide variants identified in that target region. Statistical significance $(P<0.05)$ of bias for transition over transversion mutations and mutations at WRCY motifs was calculated using a Pearson's Chi-squared test. Statistical significance of differences between proportions was calculated using a "N-1" Chi-squared test.

\section{Immunohistochemistry}

Immunohistochemistry for phosphorylated extracellular signal-regulated kinase (p-ERK), phosphorylated S6 ribosomal protein (p-S6RP), p16, and phosphatase and tensin homolog protein (PTEN) was performed on selected cases to validate candidate driver gene alterations. Programmed death ligand 1 (PD-L1) staining was also performed on selected cases. Pressure cooker antigen retrieval was performed (Target Retrieval Solution, pH 6.1; Dako, Carpinteria, CA) prior to immunohistochemistry using the following antibodies and corresponding dilutions: p-ERK 1/2 (Thr202/Tyr204) (rabbit monoclonal, D13.14.4E, Cell Signaling Technology, Danvers, MA, 1:300), pS6 ribosomal protein (Ser235/Ser236) (rabbit monoclonal, D57.2.2E, Cell Signaling Technology, 1:75), p16 (mouse monoclonal, E6H4, Ventana, Oro Valley, AZ, 1:100), PTEN (rabbit monoclonal, 138G6, Cell Signaling Technology, 1:100), PU.1 (mouse monoclonal, G148-74, BD Biosciences, San Jose, CA 1:200), CD163 (mouse monoclonal, 10D6, Leica, Buffalo Grove, IL, 1:250), CD68 (mouse monoclonal, KP-1, Leica, 1:250), and lysozyme (rabbit polyclonal, Dako, 1:7500).

\section{Antigen gene rearrangement studies}

DNA was isolated from paraffin-embedded tissue using the QIAGEN QIAamp DNA Mini Kit (QIAgen 51304). Multiplex PCR was then performed on DNA using primers differentially labeled with fluorescent dyes that hybridize to conserved framework (regions 1, 2, and 3) and joining regions of the immunoglobulin heavy chain locus at 14q32.33 (IGH gene clonality assay-ABI fluorescence detection-cat\# 11010061, InVivoScribe Technologies, BIOMED-2, San Diego, CA). The PCR products were then analyzed by capillary gel electrophoresis. This analysis was only performed on selected cases (13/28) with sufficient residual DNA after next-generation sequencing.

\section{Results}

\section{Patient cohort characteristics}

The cohort included 28 patients with a mean age of 53 years (range: 12-92 years) and a female predominance (19/28; 68\% female) (Table 1). Nearly all cases were newly diagnosed
Table 1 Patient cohort characteristics

\begin{tabular}{ll}
\hline Total number of cases, $n$ & 28 \\
Age, mean & 53 years \\
Range & $12-92$ years \\
Gender, $n(\%)$ & \\
Male & $9(32)$ \\
Female & $19(68)$ \\
Anatomic site, $n(\%)$ & \\
Lymph node & $6(21)$ \\
Skin & $5(18)$ \\
Gastrointestinal tract & $3(11)$ \\
Brain & $3(11)$ \\
Bone/soft tissue & $3(11)$ \\
Liver & $1(3.5)$ \\
Omentum & $1(3.5)$ \\
Kidney & $1(3.5)$ \\
Spleen & $1(3.5)$ \\
Breast & $1(3.5)$ \\
Heart & $1(3.5)$ \\
Pleura & $1(3.5)$ \\
Salivary gland & $1(3.5)$ \\
Tumor focality, $n$ (\%) & \\
Unifocal & $19(68)$ \\
Multifocal & $9(32)$ \\
B-lymphoblastic leukemia & $6(21)$ \\
Diffuse large B cell lymphoma & $2(7)$ \\
Follicular lymphoma & $1(3.5)$ \\
Low grade B cell lymphoma, unclassified & $1(3.5)$ \\
Multicentric juvenile xanthogranuloma & $1(3.5)$ \\
\hline & $1(3.5)$ \\
\hline
\end{tabular}

primary tumors except one case that was a recurrence 8 years following primary resection (no prior chemotherapy or radiotherapy received). The included cases were from a variety of nodal $(6 ; 21 \%)$ and extranodal sites. The most frequently involved extranodal sites were skin (18\%), gastrointestinal tract $(11 \%)$, brain $(11 \%)$, and bone/soft tissue $(11 \%)$. The majority of patients presented with unifocal disease $(19 ; 68 \%)$. A subset of patients had a preceding/concurrent diagnosis of another hematologic neoplasm (Total: 6, 21\%; Blymphoblastic leukemia: 2; diffuse large B cell lymphoma: 1; follicular lymphoma: 1; low-grade B cell lymphoma, unclassified: 1, juvenile xanthogranuloma: 1). All evaluated cases (26/26) showed diffuse and strong CD163 expression, the most specific available marker of macrophage lineage; all 15 evaluated cases were also positive for PU.1 (SPI1).

\section{Histiocytic sarcoma genome complexity}

Our next-generation sequencing approach allowed us to map an average of $11,199,267$ reads per sample, at a mean 
depth of $301 \times$, with an average of $98 \%$ of the target sequences being covered by at least 30 reads. A total of 131 somatic-coding mutations were identified with a mean coding mutational burden of $3.56 / \mathrm{Mb}$ (range: $0-10.64$ ), which is relatively low when compared to most other malignant neoplasms (Fig. 1a). The mean number of singlenucleotide variants/indels and copy-number alterations per case was 4.7 (range: $0-14$ ) and 5.3 (range: 0-30), respectively. Of the 131 coding mutations, missense mutations (84\%) were the most frequent, followed by nonsense (9\%), frameshift $(6 \%)$, and in-frame insertions/deletions (1\%). Nearly half of these mutations $(48 \%$; 63/131) were classified as likely pathogenic. Among nucleotide substitutions, there was a significant enrichment of transitions over transversions $(2.2 \times, P<0.05)$. Among the 146 copynumber alterations identified, focal (1-5 genes) gains (37\%) and losses (41\%) accounted for the majority of events with occasional segmental (15\%) and wholechromosome-level (7\%) gains and losses. About 18\% (27/ 146) of these events were classified as pathogenic or recurrent. Focal copy-number loss (one or two copy loss) involving 9p21.3 was by far the most frequent copy-number alteration (Fig. 1).

\section{RAS-MAPK pathway mutations and clinical response to mitogen-activated extracellular signal-regulated kinase (MEK) inhibition in a patient with NF1- mutated histiocytic sarcoma}

Over half $(16 / 28 ; 57 \%)$ of the cases showed at least one mutation in genes involved in the classical MAPK signaling pathway, in a largely mutually exclusive manner (Figs. 2 and $3 \mathrm{a}) . M A P 2 K 1(5 / 28 ; 18 \%)$ was the most frequently mutated gene in this pathway, with most mutations occurring at the negative regulatory helix and the core kinase domains (Fig. 3b). Hotspot-activating KRAS and NRAS mutations were also observed (involving codons 12, 13 and 146), along with two gain-of-function mutations involving the SH2 domain of PTPN11. All cases with activating $K R A S$ mutations also showed concurrent evidence of copynumber gains involving $K R A S$. Loss-of-function truncating NF1 mutations and a CBL RING domain (T402_L405del) mutation were also observed. Although no canonical BRAFV600E mutations were identified in our study, one kinase-impaired $B R A F$ variant (D594N) and another variant of possible intermediate kinase activity (G469R) [14] were identified. Finally, one case also showed a TPM3-NTRK1 fusion at canonical breakpoints (intron 1 of TPM3 and intron 11 of NTRK1); this case was otherwise morphologically and immunophenotypically typical of histiocytic sarcoma. Functional activation of RAS/MAPK signaling in our cohort was further supported by strong pERK and/or pS6RP staining in nearly all evaluated cases (5/6) harboring activating mutations in this pathway (Figs. $3 \mathrm{c}$ and $4 \mathrm{a}$, supplementary table 2).

We hypothesized that targeted therapy with cobimetinib, a MEK inhibitor that targets signaling downstream of RAS, could represent a rational therapeutic approach for patients with activating mutations in this pathway. Accordingly, a 39-year-old male with a history of B-lymphoblastic leukemia (in remission) and newly diagnosed NF1-mutated histiocytic sarcoma was treated with 3 cycles of cobimetinib therapy and showed a dramatic improvement of symptoms and a radiologic partial response (Fig. 3c). Unfortunately, the patient eventually developed progressive disease while on therapy; sequencing of the tumor showed the acquisition of a $C B L$ RING domain mutation (C396R), in addition to the previously identified $N F 1$ mutation.

\section{PI3K-AKT-MTOR pathway mutations and CDKN2A alterations}

A subset of cases (21\%) also showed pathogenic mutations predicted to lead to activation of the PI3K-AKT-MTOR signaling pathway: PTEN (D24H, Q171R), MTOR (S2215Y), PIK3RI (c.1425+1G $>\mathrm{A})$, and PIK3CA (H1047L) (Fig. 1a). For example, one case of histiocytic sarcoma presenting as a rectal mass harbored a pathogenic PTEN mutation (p.D24H) located within the $\mathrm{N}$-terminal cytoplasmic localization sequence. Immunohistochemistry for pS6RP showed diffuse and strong expression consistent with PI3K/AKT/MTOR pathway activation. Moreover, PTEN showed markedly diminished cytoplasmic expression and abnormal nucleolar localization of the protein (Fig. 4a). The majority (20/25) of activating RAS-MAPK and PI3KAKT pathway mutations were identified at high allele fractions (corrected variant allele fraction $>25 \%$, mean: $42.8 \%$ ), suggesting that these driver mutations were likely present in a large fraction of tumor cells.

The tumor-suppressor $C D K N 2 A$ was the single most frequently altered gene $(13 / 28 ; 46 \%)$ in our cohort (Fig. 1), most commonly as the result of focal copy loss involving 9p21.3 (10/13; 77\%), encompassing CDKN2A, CDKN2B, and $M T A P$ genes. These findings were also validated using immunohistochemistry for $\mathrm{p} 16$ protein expression (i.e., all cases with genetic evidence of biallelic CDKN2A inactivation showed complete loss of p16 protein expression; Fig. 4b).

\section{PD-L1 expression and copy-number analysis at 9p24.1}

The majority of tested cases (8/10) showed expression of PD-L1 on neoplastic cells (defined as expression in $>5 \%$ of lesional cells). Of note, most cases (5/10) showed strong membranous staining in a large subset of lesional cells 


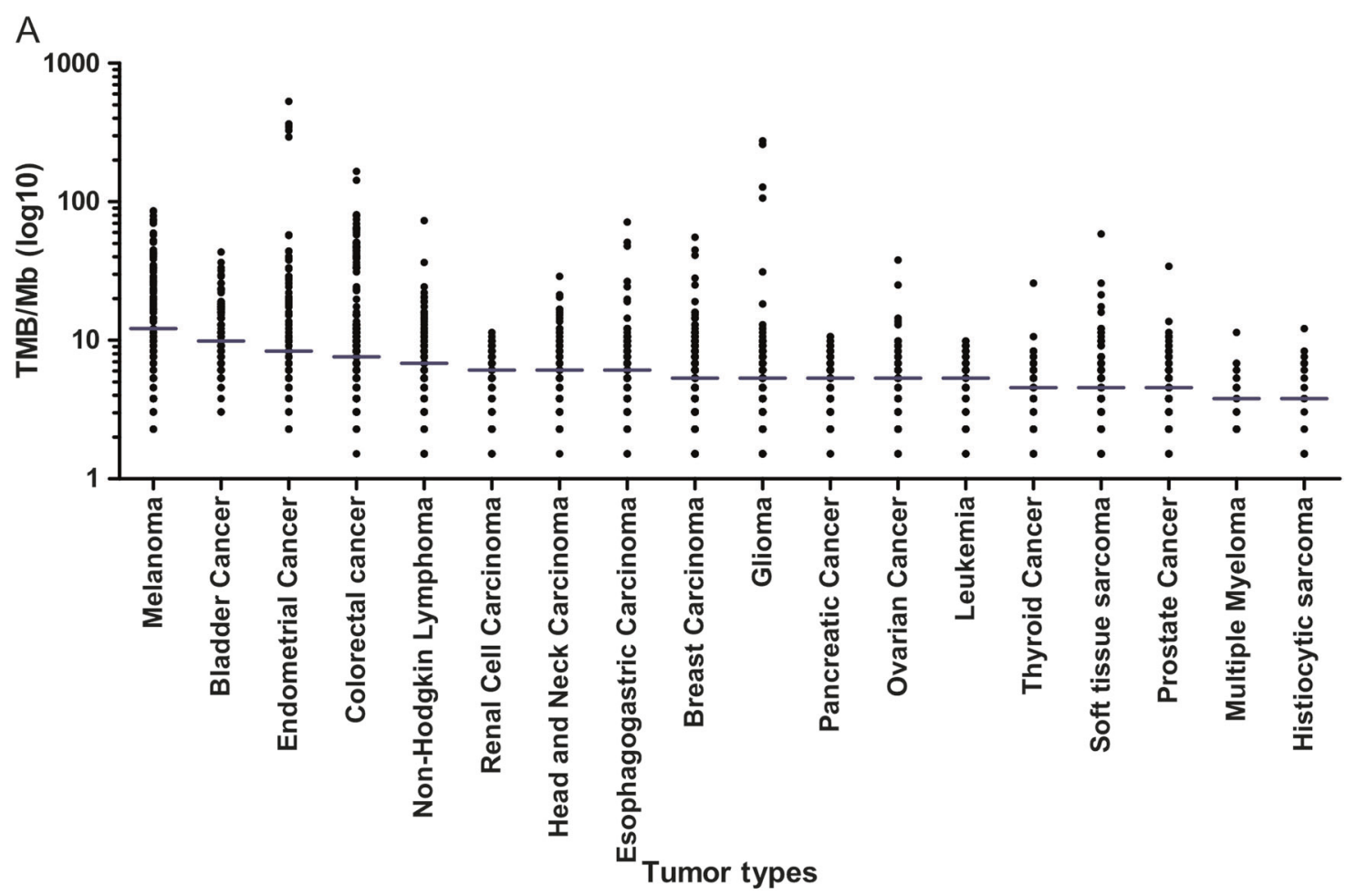

B
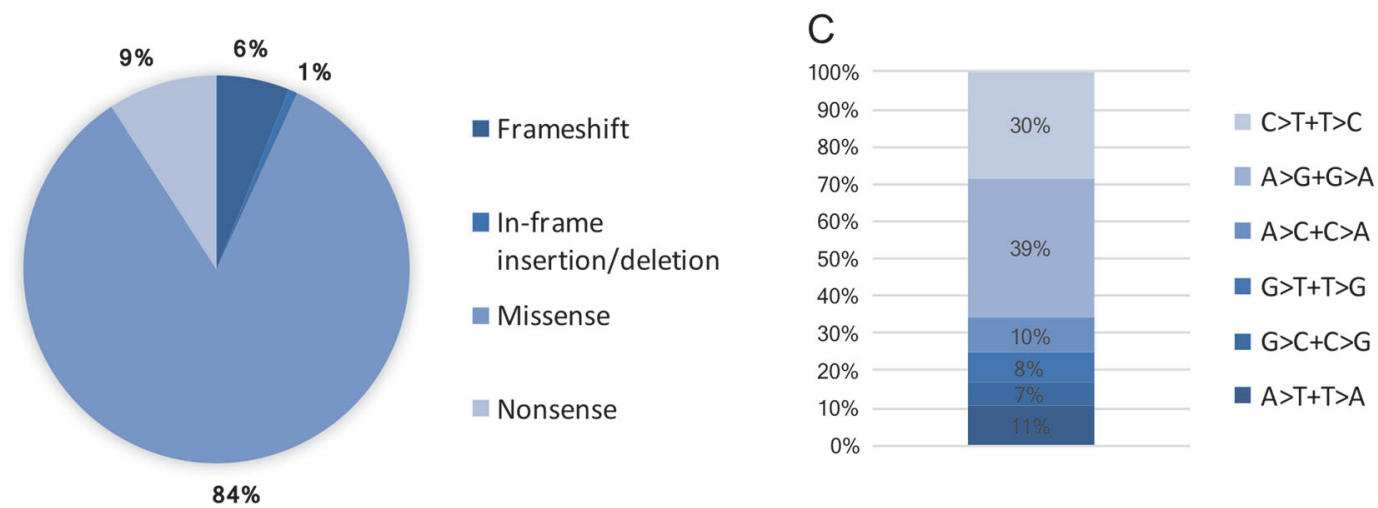

D

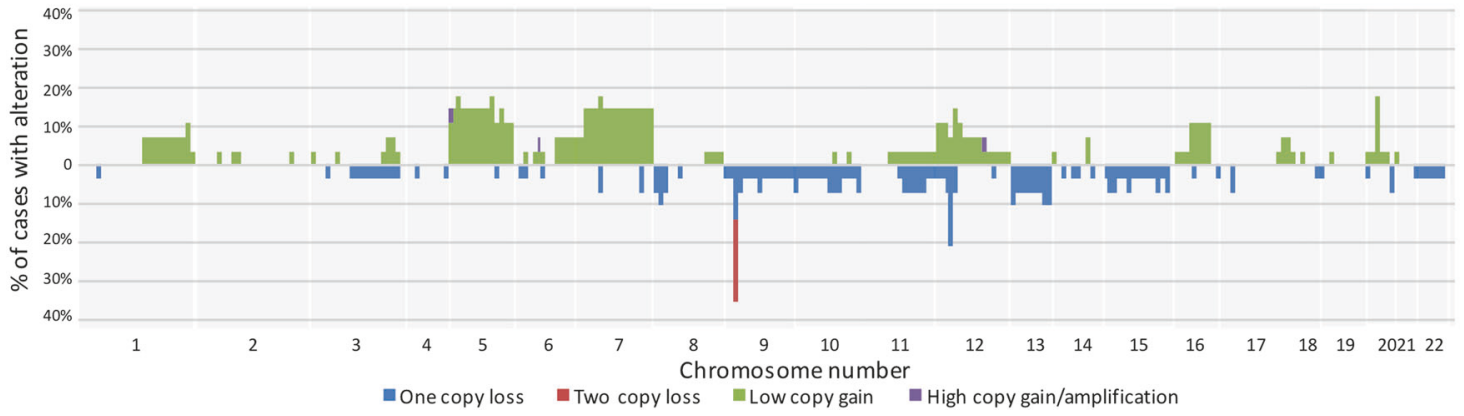

Fig. 1 Coding genome complexity of histiocytic sarcoma. a Tumor mutational burden (TMB, number of coding mutations/Mb) of histiocytic sarcoma and other tumor types. Data on other tumor types obtained from DFCI/BWH PROFILE cohort data (using internal instance of cBioportal $[43,44])$. b Classification of coding mutations. $\mathbf{c}$ Pattern of nucleotide substitutions. d Stacked histogram showing frequency of genome-wide copy-number alterations across the cohort 


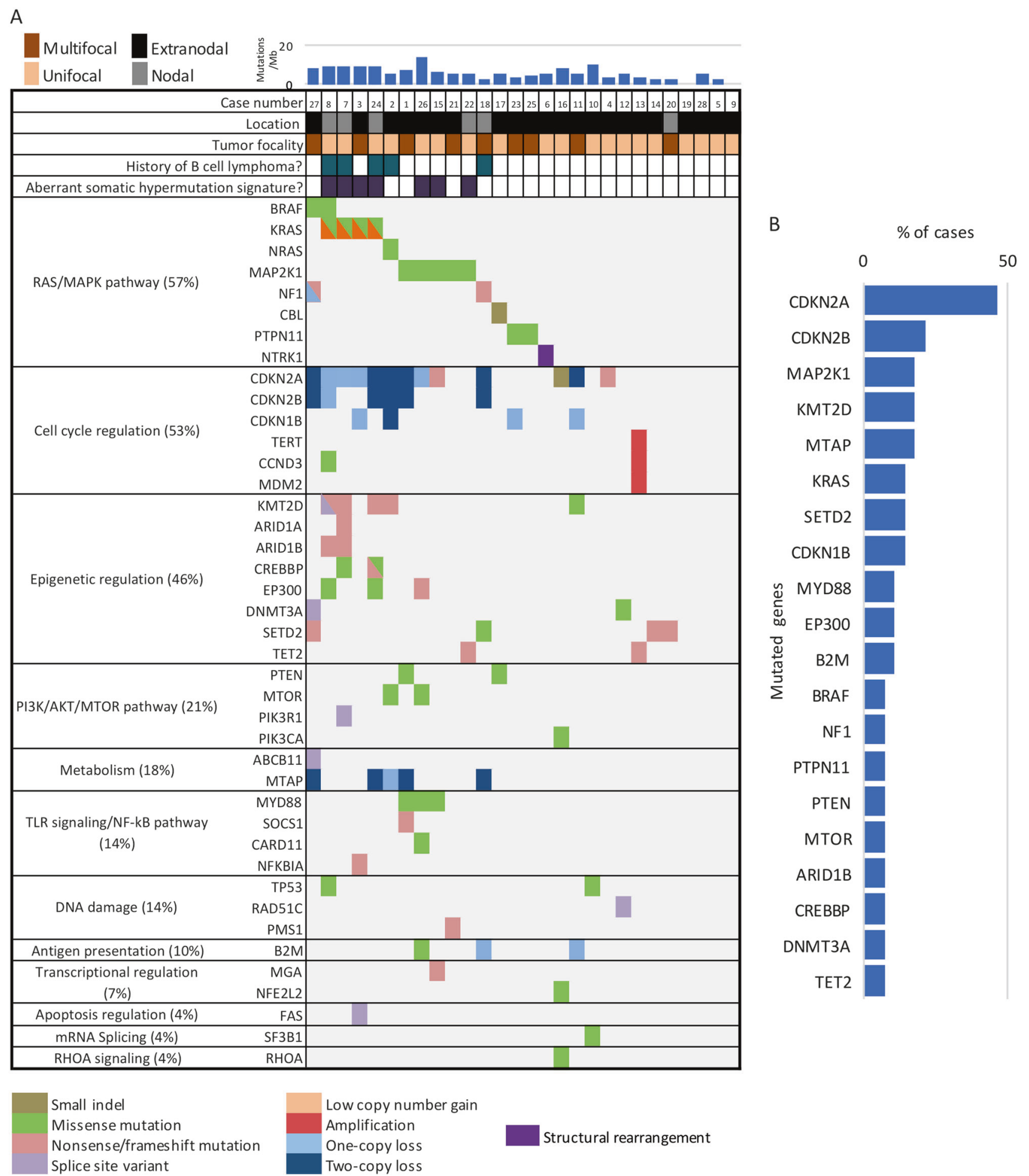

Fig. 2 Mutational landscape of histiocytic sarcoma. a Summary table of all pathogenic variants (single-nucleotide variants, small insertions/ deletions, copy number, and structural alterations) identified in 28 cases of histiocytic sarcoma grouped by pathway (likely singlenucleotide polymorphisms and variants of uncertain significance excluded). Each column represents data for a single patient. Relevant clinical data (location, tumor focality, history of B cell lymphoma) and the presence/absence of an aberrant somatic hypermutation signature are also provided for each case. The colored cells indicate the presence of a history of B cell lymphoma and an aberrant somatic hypermutation signature. The histogram on top shows tumor mutational burden (number of coding mutations/megabase). b Genes arranged in decreasing order of mutation frequency (top 20) 
A

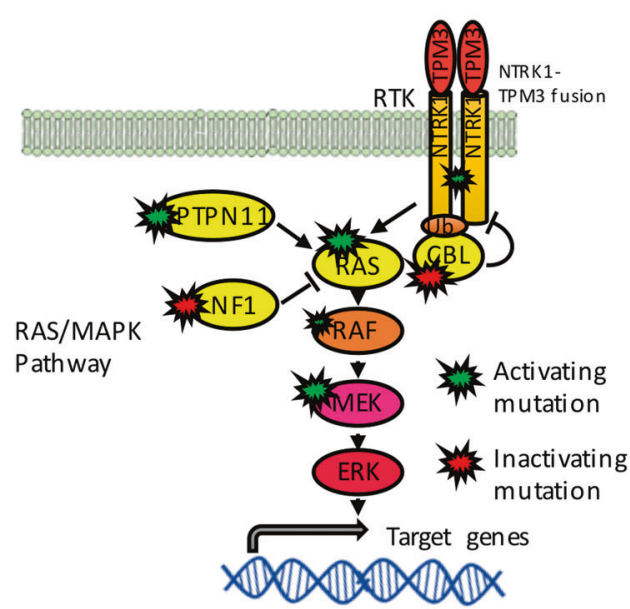

B

BRAF
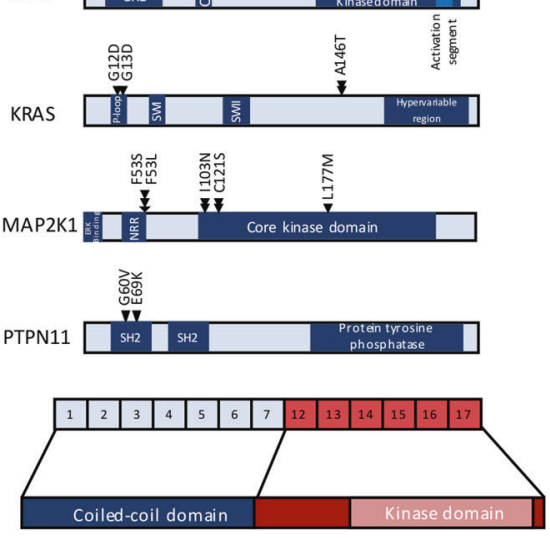

\begin{tabular}{l|l|l|}
\hline TPM3 & NTRK1 \\
\hline
\end{tabular}

C

Achieved
B-ALL complete diagnosis remission
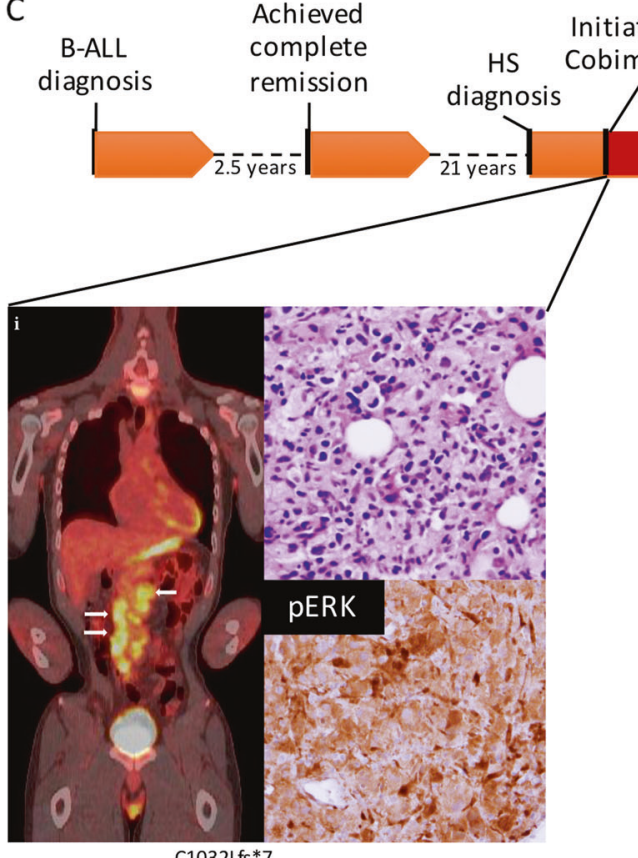

C10321f**7

RasGAP GRD

NF1

$\begin{array}{ccc}\text { 3itiation of } & 3 \text { cycles } & 5 \text { cycles } \\ \text { Partial } & \text { Disease } \\ \text { response } & \text { progression }\end{array}$

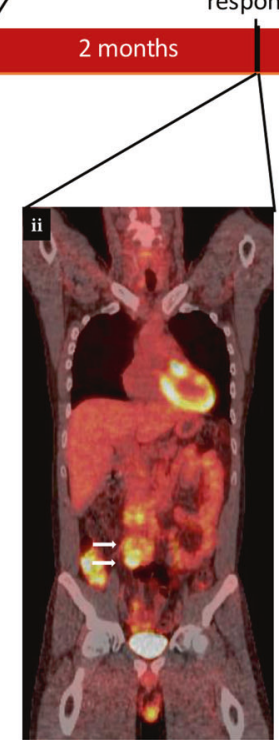

3 months

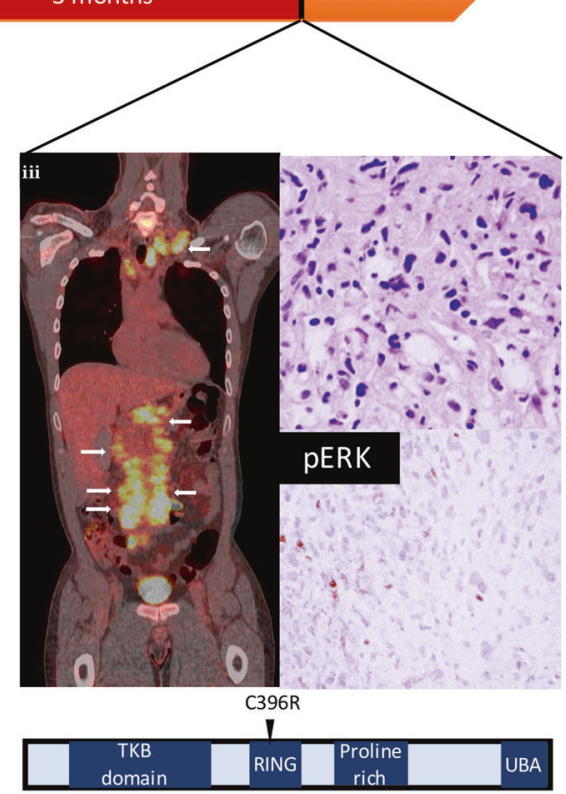

CBL

D

\begin{tabular}{|c|c|c|c|c|c|}
\hline Time point & $\begin{array}{l}\text { Tumor } \\
\text { cellularity }\end{array}$ & Gene & Mutation & Allele frequency & Coverage \\
\hline \multirow{2}{*}{ Initial diagnosis } & \multirow{2}{*}{$80 \%$} & $\mathrm{NF1}$ & c.3090_3091ins T (p.C1032Lfs*7), exon 23 & $28 \%$ & 220 \\
\hline & & SETD2 & $c .4874 G>A(p . R 1625 H)$, exon 7 & $47 \%$ & 218 \\
\hline \multirow{3}{*}{$\begin{array}{c}\text { Disease progression } \\
\text { on cobimetinib }\end{array}$} & \multirow{3}{*}{$80 \%$} & CBL & c.1186T>C (p.C396R), exon 8 & $22 \%$ & 280 \\
\hline & & NF1 & c.3090_3091ins T (p.C1032Lfs ${ }^{*} 7$ ), exon 23 & $33 \%$ & 281 \\
\hline & & SETD2 & $c .4874 G>A(p . R 1625 H)$, exon 7 & $56 \%$ & 243 \\
\hline
\end{tabular}


Fig. 3 RAS/MAPK pathway alterations in histiocytic sarcoma and response to targeted therapy in a patient with $N F 1$-mutated histiocytic sarcoma. a Summary of activating mutations involving the RAS/ MAPK signaling pathway. b Schematic showing coding mutations of the most frequently mutated genes of the RAS/MAPK signaling pathway including a TPM3-NTRK1 fusion between exons 1-7 of TPM3 (coiled-coil domain) and exons 12-17 of NTRK1 (includes the kinase domain). c Clinical timeline of events with corresponding imaging, histopathologic, and molecular findings. (i) Baseline coronal FDG-PET/CT showing intensely FDG-avid retroperitoneal and mesenteric lymphadenopathy (arrows). Biopsy of the mesenteric lymph node showed sheets of large atypical histiocytoid cells (Hematoxylin \& Eosin stain, $\times 400$ magnification) with highly pleomorphic forms and giant cells. A NF1-truncating mutation was identified at diagnosis (p.C1032Lfs*7), which would result in loss of the major catalytic GAP-related domain (GRD) (schematic below images), (ii) Partial metabolic response after three cycles of cobimetinib with residual FDG-avid mesenteric lymph nodes (arrows), (iii) Disease progression after five cycles with increased mesenteric and retroperitoneal adenopathy (lower arrows) as well as new FDG-avid supraclavicular and mediastinal adenopathy (upper arrows). A biopsy of the same mesenteric lymph node at disease progression showed slightly decreased tumor cellularity with stromal hyalinization $(\times 400$ magnification). There was also dramatic reduction in phosphorylated ERK expression on cobimetinib therapy consistent with drug-mediated MEK inhibition ( $\times 400$ magnification); however, there was persistent expression of phosphorylated S6 protein (data not shown). This was also associated with the acquisition of a $C B L$ RING domain mutation (p.C396R) (schematic below images). d Table showing mutations and respective allele fractions at diagnosis and at disease progression following therapy

(>20\%). Expression of PD-L1 was also observed in normal macrophages (internal control) with no significant differences in staining intensity in tumor cells relative to macrophages. None of these cases showed evidence of copynumber gain involving 9p24.1 (which includes CD274/PDL1; Fig. 5).

\section{Molecular genetic overlap with B cell neoplasms: further evidence for a clonal relationship}

A subset of cases $(7 / 28 ; 25 \%)$ showed a mutational signature of "aberrant somatic hypermutation" in genes that are known to be targets of this process (BCL6, BCL2, CIITA, $M Y C$, SOCS1, PAX5) [15] (Fig. 6a-c). Further, this signature was more frequently seen in tumors of patients with a history of B cell lymphoma $(3 / 7,43 \%$ vs $2 / 21,9 \%$; $P<$ $0.05)$. Cases with aberrant somatic hypermutation also more frequently $(6 / 7,86 \%$ vs $3 / 21,14 \% ; P<0.05)$ showed comutations in genes involved in Toll-like receptor (TLR)/ nuclear factor (NF)- $\kappa \mathrm{B}$ signaling and epigenetic regulation (e.g., KMT2D), which are frequently identified in B cell lymphomas (Fig. 6d). Interestingly, we also observed a significant association between the presence of RAS-MAPK and/or $C D K N 2 A$ mutations and the presence of an aberrant somatic hypermutation signature $(P<0.05)$ or a history of $\mathrm{B}$ cell lymphoma $(P<0.05)$. Finally, 5 of the 13 tested cases
(39\%) showed a clonal immunoglobulin heavy chain gene rearrangement (Fig. 6d).

\section{Discussion}

Our study provides the first detailed description of the coding genome of human histiocytic sarcoma using a targeted next-generation sequencing approach on a large cohort of well-characterized cases $(N=28)$. The mean somatic coding mutational burden was low (3.56/Mb) when compared to other epithelial and non-epithelial malignancies. A pathogenic driver genetic alteration was identified in the vast majority of cases $(86 \% ; 24 / 28)$. Of note, nearly all genes with pathogenic mutations identified in this study were covered by both versions of the sequencing panel used (36/41). Our findings significantly expand the current understanding of the pathogenesis of histiocytic sarcoma and offer new opportunities for the use of rational targeted therapies to treat this disease.

We have identified recurrent activating genetic alterations involving the classical MAPK signaling pathway in the majority of cases $(16 / 28 ; 57 \%)$. Individual case reports and small case series have described oncogenic-activating mutations involving the RAS/RAF/MEK/ERK pathway including BRAF (V600E, G464V, G466R, F595L) [16-18], KRAS (Q61H) [16], PTPN11 (E72K, G503V) [16, 19], and $M A P 2 K 1[17,20]$ in histiocytic sarcoma. However, this is the first large series demonstrating recurrent mutations involving the RAS/MAPK pathway in a large subset of histiocytic sarcoma cases. In our series, activating MAP2K1 mutations involving the negative regulatory helix and the core kinase domains were most frequent. These gain-offunction mutations have been previously described in a number of other tumor types, including histiocytic neoplasms [2, 21, 22]. Hotspot-activating KRAS mutations were also identified in four cases, all of which, interestingly, showed concurrent KRAS copy gain. This is a welldescribed phenomenon in other solid tumors such as lung adenocarcinoma, where mutant KRAS copy gain is associated with advanced disease and a distinct metabolic program with unique therapeutic vulnerabilities in vitro and in vivo [23]. The two likely activating mutations involving the SH2 domain of PTPN11 have been described as recurrent in juvenile myelomonocytic leukemia and other myeloid neoplasms [24]. Further, one previous study [16] reported an activating PTPN11 tyrosine phosphatase domain mutation (p.G503V) in a case of histiocytic sarcoma. Although no canonical BRAFV600E mutations were identified in our study, one kinase-impaired $B R A F$ variant (D594N) and another variant of possible intermediate kinase activity (G469R) [14] were identified. These lowactivity $B R A F$ mutants have been shown to drive MEK/ 

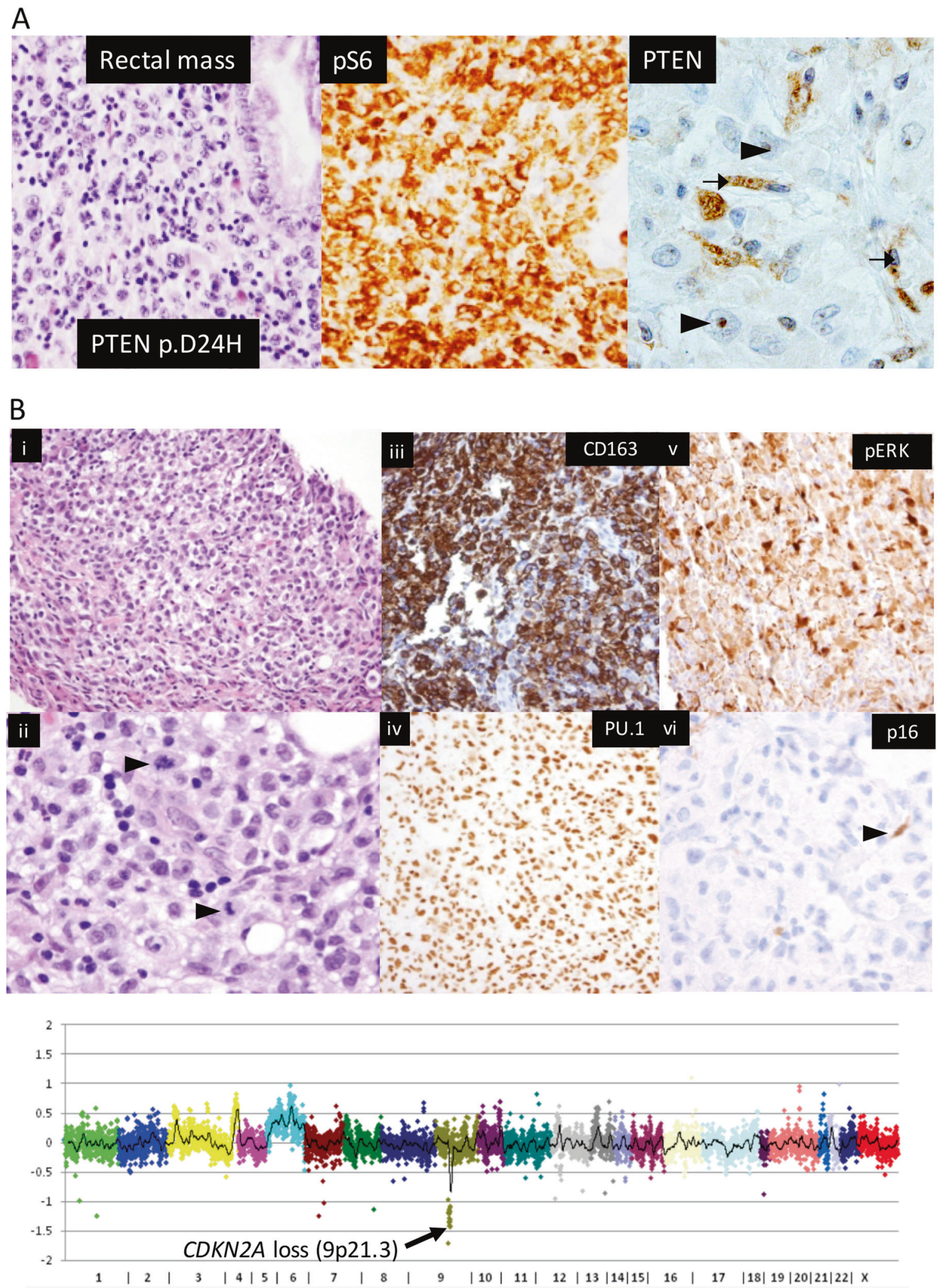

ERK signaling in a RAS-dependent manner and frequently coexist with loss-of-function NF1 mutations or activating $R A S$ mutations [14, 18], as was observed in our study. This co-mutation pattern has also been previously described in histiocytic sarcoma [16, 18]. One case also harbored a TPM3-NTRK1 rearrangement, resulting in fusion of the $\mathrm{N}$ - 
Fig. 4 Immunohistochemical validation of candidate driver genes. a A case of histiocytic sarcoma (i-H\&E x400 magnification, ii-H\&E x1000 magnification; arrowheads show mitotic figures; iii-iv-immunohistochemistry $\mathrm{x} 400$ magnification) harboring a NF1 loss-of-function mutation and two-copy deletion involving 9p21.3 including CDKN2A (lower). The tumor showed diffuse and strong expression of p-ERK ( $\mathrm{v}-$ x400 magnification). Immunohistochemistry for p16 demonstrated loss of staining in tumor cells (arrowheads show intact expression of p16 in background inflammatory cells; vi-x1000 magnification). b A case of histiocytic sarcoma (H\&E x400 magnification) presenting as a rectal mass with PTEN mutation involving the $\mathrm{N}$-terminal cytoplasmic localization sequence. Immunohistochemistry for p-S6 protein showed diffuse and strong expression (x400 magnification). Immunohistochemistry for PTEN (x1000 magnification) showed loss of cytoplasmic protein expression in tumor cells and abnormal nucleolar localization (arrowheads). Note intact expression of PTEN in background stromal and inflammatory cells (arrow)

terminal coiled-coil domain of TPM3 to the C-terminal kinase domain of NTRK1. This likely results in constitutive dimerization and ligand-independent activation of the NTRK1 kinase domain, which is sensitive to TRKA kinase inhibition [25]. NTRK fusions can be identified in a wide range of malignancies and can be targeted with a potent TRK small molecular inhibitor to produce marked and durable responses, regardless of patient age or tumor type [26].

There are also published case reports of demonstrable responses to drugs targeting the RAS-MAPK pathway. Recently, Gounder et al. [20] demonstrated a durable complete response ( $>2$ years) to trametinib in a patient with MAP2K1-mutated (p.F53L) histiocytic sarcoma. Voruz et al. [19] described a case of multifocal histiocytic sarcoma harboring a PTPN11 (p.E72K) mutation, which showed partial response to MEK inhibition by trametinib. Idbaih et al. [27] treated a patient with isolated central nervous system histiocytic sarcoma harboring a BRAF p.V600E mutation with vemurafenib who had a dramatic clinical and radiologic response. In the latter two reports, the tumors eventually became refractory to targeted therapy and a potential mechanism of resistance was not explored. In our patient, we identified an oncogenic $C B L$ RING finger mutation that emerged at the time of disease progression, which might lead to resistance by promoting escape from MEK dependence by broad downstream activation of alternative signaling pathways, such as PI3K/AKT signaling via loss of ubiquitin/proteasome-mediated degradation of diverse tyrosine kinases. There is emerging in vivo and in vitro evidence from studying $C B L$-mutated myeloid neoplasms that RING finger mutations result in sustained Akt pro-survival signaling [28, 29]. There is also a reported case of a $C B L$ RING domain mutation (C384R) potentially driving resistance to third-generation tyrosine kinase inhibitors in lung adenocarcinoma [30].
A subset of cases also showed activating mutations involving the PI3K-AKT-MTOR signaling pathway. These include known hotspot kinase-activating mutations involving MTOR (S2215Y) and PIK3CA (H1047L). Activating PIK3CA mutations have been identified previously in one case of histiocytic sarcoma [16] and cases of $B R A F$-wild-type Erdheim-Chester disease [2]. We also identified a relatively uncommon inactivating PTEN (p. D24H) mutation, located within the N-terminal cytoplasmic localization sequence, which has been shown to result in abnormal nuclear sequestration and loss of cytoplasmic localization, resulting in impaired growthsuppressive properties of PTEN [31]. Consistent with this model, immunohistochemistry for PTEN showed markedly diminished cytoplasmic expression and abnormal nucleolar localization and pS6RP showed strong expression, supporting functional PI3K pathway activation. We also identified a splice donor site mutation involving PIK3R1 (c.1425+1G $>$ A), which has been shown to result in exon 11 skipping, loss of the inter-SH2 domain, and hyperactive, constitutive PI3K signaling. This mutation has been identified in patients with immunodeficiency disorders but not previously as a somatic mutation in cancer [32]. Of note, the activating RAS-MAPK and PI3K-AKT pathway mutations were identified at relatively high allele fractions, suggesting that these mutations are present in a large fraction of tumor cells and are likely drivers of tumor biology.

The $C D K N 2 A$ tumor-suppressor gene was the most frequently altered gene, by mutation or deletion. The most frequent mechanism of $C D K N 2 A$ inactivation was through focal deletion at $9 \mathrm{p} 21.3$, which may or may not include the nearby genes $C D K N 2 B$ and MTAP. Among cases with focal 9 p21.3 deletion, over half showed evidence of biallelic deletion. Moreover, immunohistochemistry for p16 was also a useful surrogate marker for $C D K N 2 A$ deletion (100\% concordance in 8 cases examined). The CDKN2A gene encodes for two proteins, p16(INK4) and p14(ARF), through shared coding regions and alternate reading frames. These proteins function as a cyclin-dependent kinase inhibitor and stabilize p53 levels through MDM2 binding, respectively. Therefore, $C D K N 2 A$ is an important tumorsuppressor gene that is inactivated frequently in a variety of human malignancies [33]. Homozygous CDKN2A deletion has been previously demonstrated in a case of histiocytic sarcoma arising through clonal evolution of Blymphoblastic leukemia [34]. One study has also shown that $C D K N 2 A$ and PTEN double mutant mice [35] have a dramatically increased incidence of histiocytic sarcoma. In keeping with this, we observed frequent mutations involved PI3K-AKT-MTOR and cyclin D-CDK4/6-INK4-Rb pathways and also a trend (not statistically significant) toward the co-occurrence of mutations involving these pathways, 
further supporting a model of cooperative interaction between these pathways in the pathogenesis of histiocytic sarcoma.

We also describe frequent, albeit variable, PD-L1 expression in the lesional cells of histiocytic sarcoma, as has been previously described [36, 37]. Since PD-L1 is also expressed in normal macrophages [37], this finding may simply represent further evidence of macrophage lineage in this tumor. We did not identify a clear structural genetic mechanism for PD-L1 expression in histiocytic sarcoma, as described in other hematologic malignancies [38]. Expression of PD-L1 is associated with a suppressive immune microenvironment in BRAFV600E-mutated Langerhans cell histiocytosis [39]; its biologic significance in other histiocytic neoplasms is unclear. The utility of PD-L1/PD-1 blockade in histiocytic neoplasms is currently unknown but is being explored in a clinical trial (NCT03316573).
A

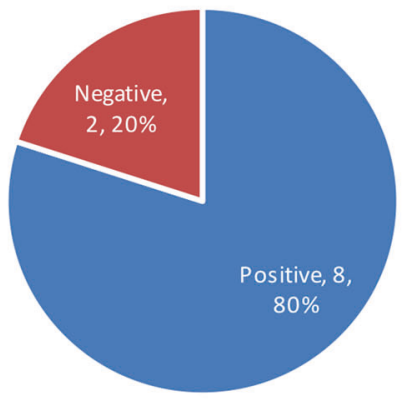

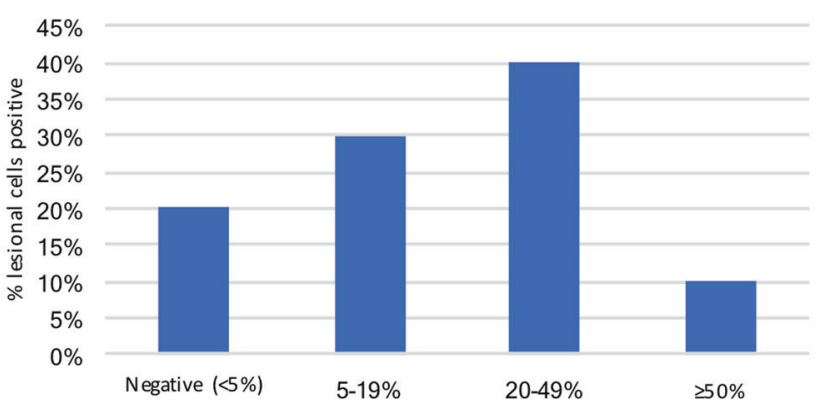

B

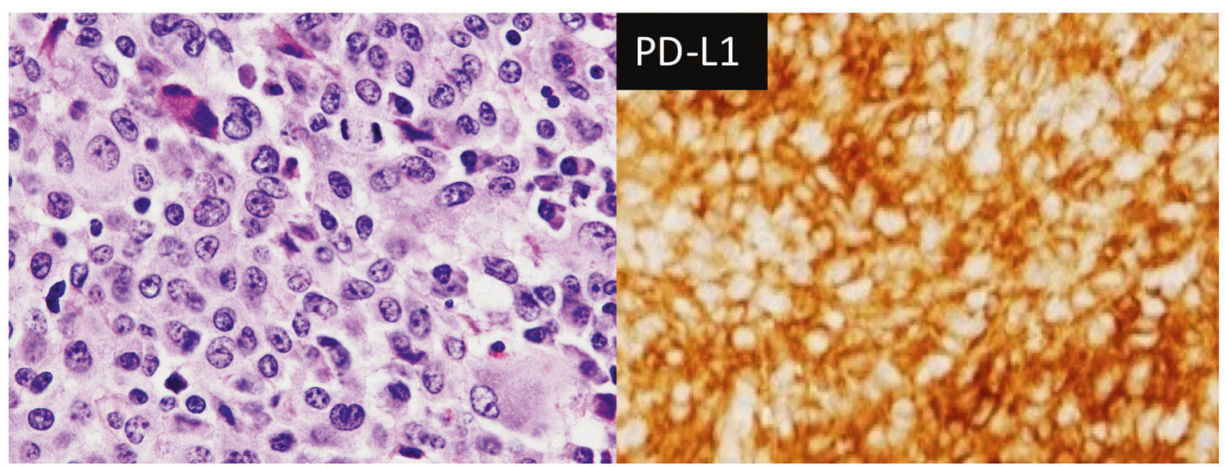

C

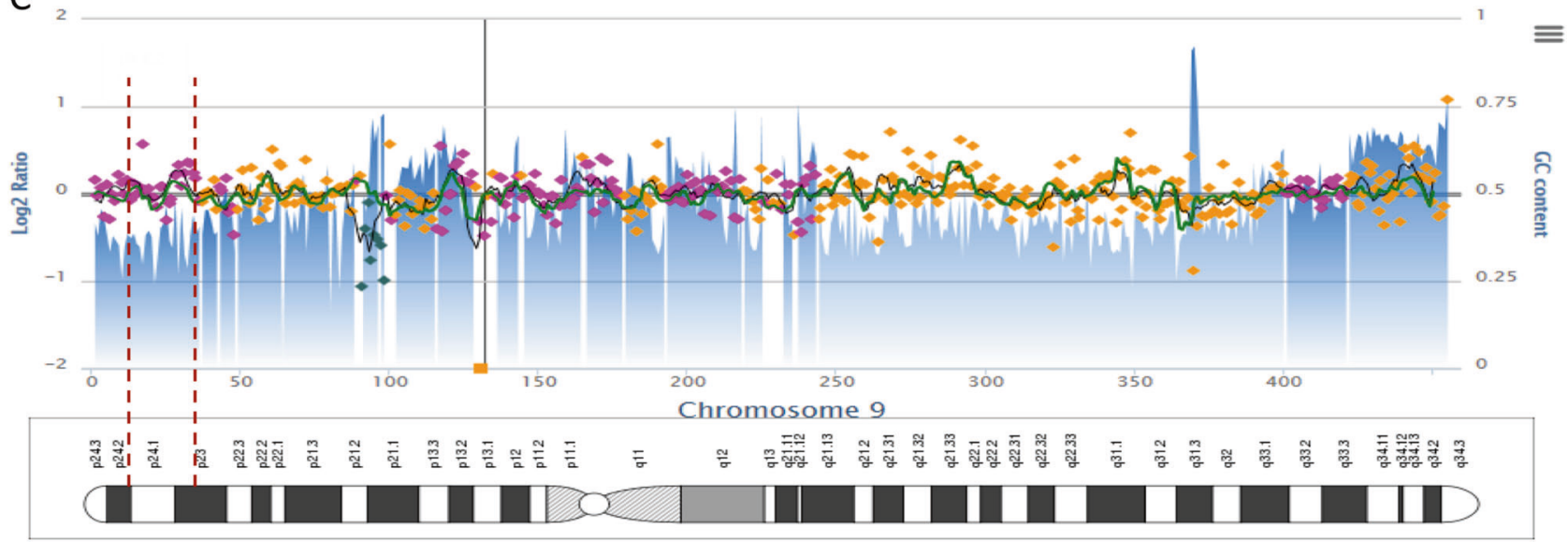

Fig. 5 Frequent expression of PD-L1 in histiocytic sarcoma is not associated with gains of 9p24. a Pie chart and histogram showing frequency and extent (percentage of lesional cells positive) of PD-L1 protein expression, respectively, in 10 cases of histiocytic sarcoma. b Case of histiocytic sarcoma presenting as a parotid mass (left, $\mathrm{H} \& \mathrm{E}$ x400 magnification) showing diffuse and strong membranous reactivity for PD-L1 (right, x400 magnification) but c no evidence of copynumber changes at 9p24.1 (red dotted lines show area between 9p23 and $9 \mathrm{p} 24.2)$ 


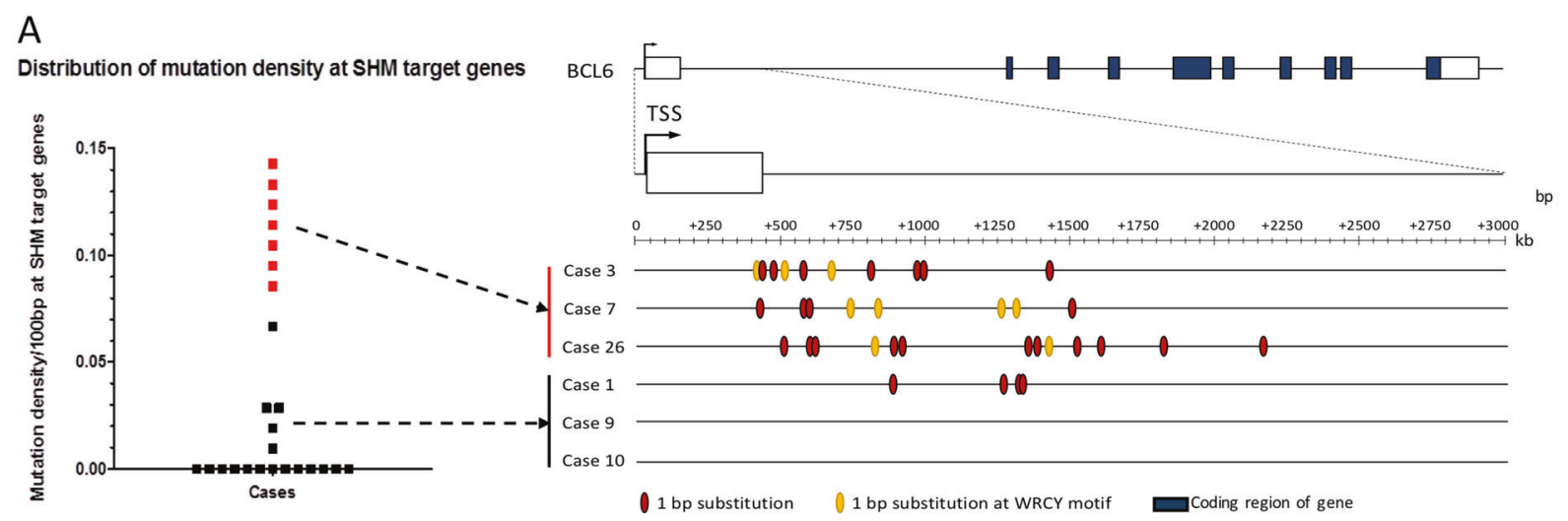

B

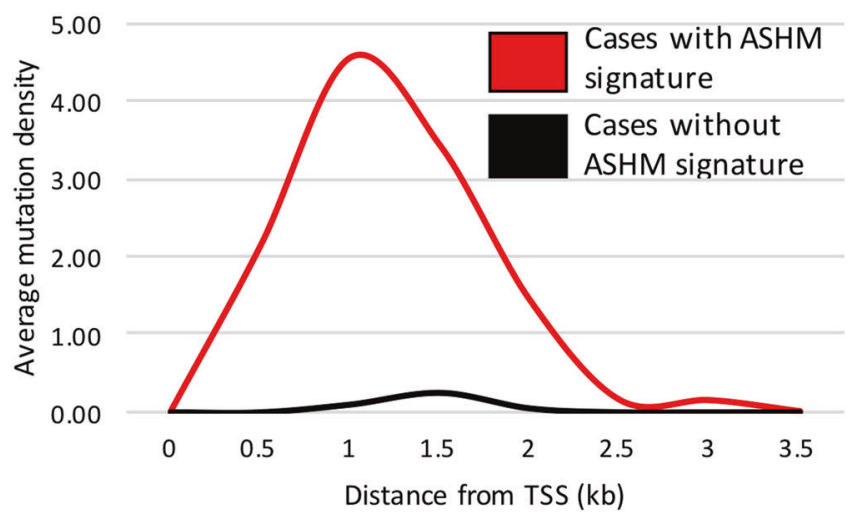

C

\begin{tabular}{|l|l|}
\hline Mutation density at ASHM targets/100bp & 0.114 \\
\hline Transitions/transversions ( $p$-value) & $1.15(p<0.001)$ \\
\hline Single nucleotide substitutions & 84 \\
\hline Insertions/deletions & 0 \\
\hline Mutation density at WRCY motifs/100bp & 0.035 \\
\hline$\%$ mutations at WRCY motifs & $31 \%$ \\
\hline WRCY motif bias (p-value) & $1.30(p<0.05)$ \\
\hline Median location from TSS & $921.5 \mathrm{bp}$ \\
\hline
\end{tabular}

SHM: somatic hypermutation; TSS: transcription start site

D

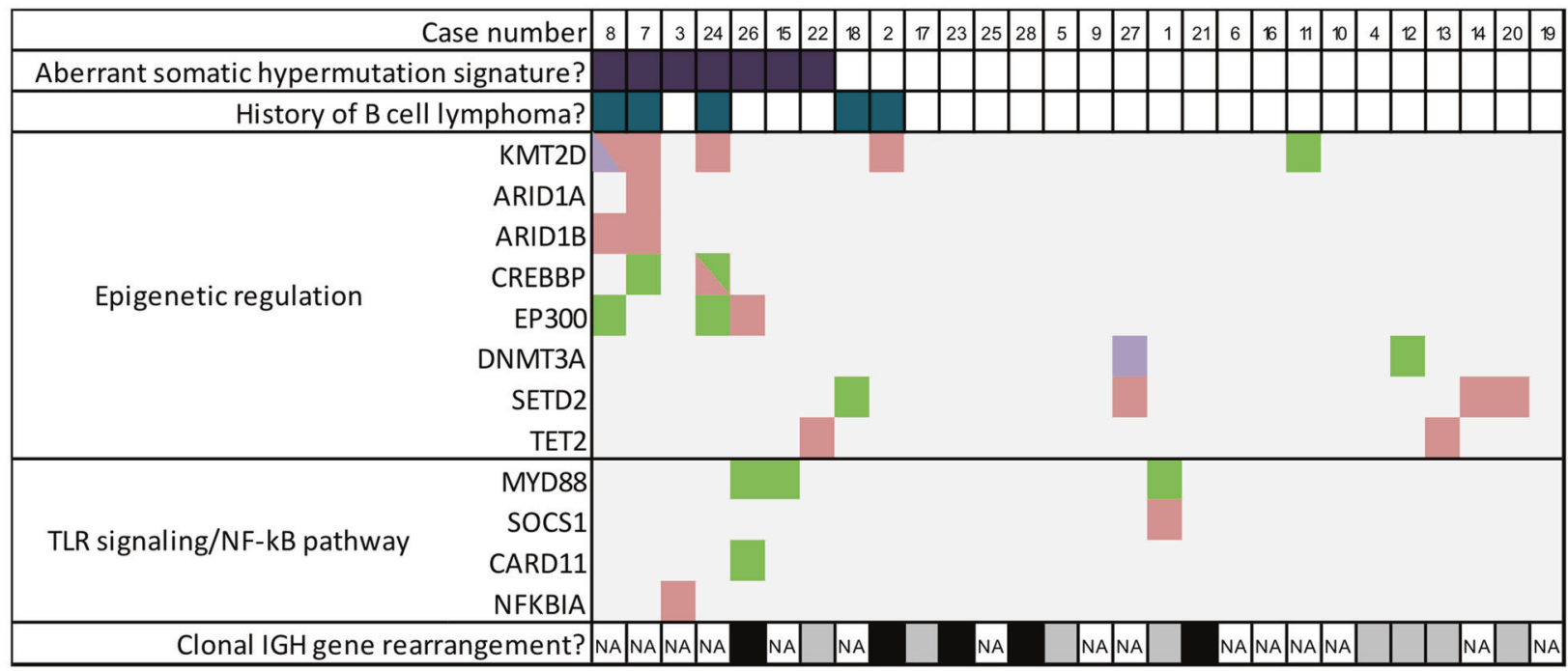

\section{Polyclonal Clonal}

Fig. 6 Evidence for aberrant somatic hypermutation in histiocytic sarcomas. a A subset of cases (red squares, 7/28) showing high mutation frequency at target genes of aberrant somatic hypermutation; (right) location and characteristics of mutations involving BCL6. b Distribution of somatic mutations involving target genes of somatic hypermutation as a function of distance from the transcription start site (TSS). Average mutation density indicates the group mean of the average number of mutations per case within overlapping intervals of
Missense mutation Nonsense/frameshift mutation Splice site variant

NA DNA not available

500 bp. c Summary characteristics of mutations involving target genes of somatic hypermutation. d Molecular genetic overlap between a subset of histiocytic sarcomas and B cell lymphomas. Each column represents a single case of histiocytic sarcoma with combined mutation data in selected genes recurrently mutated in B cell lymphomas, the presence of an aberrant somatic hypermutation signature, documented history of B cell lymphoma, and the presence of a clonal $I G H$ gene rearrangement 
Finally, in a subset of cases (25\%), we also identified a mutational signature that resembles aberrant somatic hypermutation. Aberrant somatic hypermutation is a consequence of mis-targeting of somatic hypermutation machinery to proto-oncogenes instead of immunoglobulin genes in B cell-derived tumors, such as diffuse large B cell lymphoma [13, 40]. Interestingly, in our series, this group was enriched for patients with a history of B cell lymphoma and tumors with co-mutations in genes that are frequently mutated in B cell neoplasms (TLR/NF- $\mathrm{KB}$ signaling and epigenetic regulation). These findings suggest molecular genetic overlap between histiocytic sarcoma and B cell neoplasms. Previous studies have demonstrated a clear clonal relationship between follicular lymphoma [7], chronic lymphocytic leukemia [6] and other hematologic malignancies [41] and histiocytic/dendritic cell neoplasms using comparative sequencing/PCR of immunoglobulin genes and/or cytogenetic (e.g., fluorescence in situ hybridization) methods. In addition, Chen et al. [42] observed that nearly half of all sporadic cases (unrelated to a preexisting B cell neoplasm) of histiocytic/dendritic cell sarcomas can show clonal rearrangements of immunoglobulin genes. We have confirmed this result in our cohort, where we observed clonal immunoglobulin heavy chain gene rearrangements in a large subset ( 40\%) of evaluated cases. There was no statistically significant relationship between the presence of clonal immunoglobulin gene rearrangements and history of lymphoma or the presence of an aberrant somatic hypermutation signature, but this is likely limited by small sample sizes (insufficient DNA for PCR analysis). Finally, we also observed that cases showing an aberrant somatic hypermutation signature and patients with a history of B cell lymphoma more frequently showed RASMAPK mutations and/or CDKN2A alterations. Overall, these findings further support the prevailing notion that a subset of histiocytic sarcomas arise as a result of clonal evolution from pre-existing B cell neoplasms or B cell lineage-committed precursors with a possible role for RAS/ MAPK and/or CDKN2A mutations in orchestrating this transformation. However, one major limitation of our study is the lack of comparative genetic analysis of histiocytic sarcoma and preceding B cell lymphoma in the same patient to distinguish cases of true "secondary" histiocytic sarcoma arising out of clonal evolution of B cell malignancies from "primary" histiocytic sarcomas.

In summary, we have identified diverse and activating RAS-MAPK and PI3K-AKT-MTOR pathway mutations and $C D K N 2 A$ tumor-suppressor gene alterations in the majority of histiocytic sarcomas. Further, a subset of cases harboring these mutations also show striking genetic similarities with B cell lymphomas. These findings support a model of cooperative interaction between RAS-MAPK, PI3K-AKT-MTOR, and cyclin D-CDK4/6-INK4-Rb pathways in histiocytic sarcoma and clonal evolution from B cell neoplasia, providing a rational basis for the targeting of these pathways in the treatment of this aggressive disease.

Acknowledgements Funding for this study was provided by the Department of Pathology and the Center for Advanced Molecular Diagnostics at Brigham and Women's Hospital. The authors would like to thank all the technical staff of the Center for Advanced Molecular Diagnostics and immunohistochemistry laboratory at the Brigham and Women's Hospital for their contributions. In particular, we are grateful to Michele Baltay and Mei Zheng for their invaluable help with this study. We also thank Dr. Jyothi Jagannathan for providing representative images of radiologic studies.

\section{Compliance with ethical standards}

Conflict of interest GKG is a consultant for Moderna Therapeutics. EDJ is a consultant for Astra-Zeneca, Seattle Genetics, and Merck. LMS is a consultant for Foghorn Therapeutics. JLH is a consultant for Eli Lilly and Epizyme. The other authors declare that they have no conflict of interest.

Publisher's note: Springer Nature remains neutral with regard to jurisdictional claims in published maps and institutional affiliations.

\section{References}

1. Badalian-Very G, Vergilio J-A, Degar BA, et al. Recurrent BRAF mutations in Langerhans cell histiocytosis. Blood. 2010;116:1919-23.

2. Diamond EL, Durham BH, Haroche J, et al. Diverse and targetable kinase alterations drive histiocytic neoplasms. Cancer Discov. 2016;6:154-65.

3. Hyman DM, Puzanov I, Subbiah V, et al. Vemurafenib in multiple nonmelanoma cancers with BRAF V600 mutations. N Engl J Med. 2015;373:726-36.

4. Haroche J, Cohen-Aubart F, Emile JF, et al. Reproducible and sustained efficacy of targeted therapy with vemurafenib in patients with BRAF(V600E)-mutated Erdheim-Chester disease. J Clin Oncol. 2015;33:411-8.

5. Hornick JL, Jaffe ES, Fletcher CDM. Extranodal histiocytic sarcoma: clinicopathologic analysis of 14 cases of a rare epithelioid malignancy. Am J Surg Pathol. 2004;28:1133-44.

6. Shao H, Xi L, Raffeld M, et al. Clonally related histiocytic/dendritic cell sarcoma and chronic lymphocytic leukemia/small lymphocytic lymphoma: a study of seven cases. Mod Pathol. 2011;24:1421-32.

7. Feldman AL, Arber DA, Pittaluga S, et al. Clonally related follicular lymphomas and histiocytic/dendritic cell sarcomas: evidence for transdifferentiation of the follicular lymphoma clone. Blood. 2008;111:5433-9.

8. Gounder M, Desai V, Kuk D, et al. Impact of surgery, radiation and systemic therapy on the outcomes of patients with dendritic cell and histiocytic sarcomas. Eur J Cancer. 2015;51:2413-22.

9. Sholl LM, Do K, Shivdasani P, et al. Institutional implementation of clinical tumor profiling on an unselected cancer population. JCI Insight. 2016;1:e87062.

10. McKenna A, Hanna M, Banks E, et al. The Genome Analysis Toolkit: a MapReduce framework for analyzing next-generation DNA sequencing data. Genome Res. 2010;20:1297-303.

11. Cibulskis K, Lawrence MS, Carter SL, et al. Sensitive detection of somatic point mutations in impure and heterogeneous cancer samples. Nat Biotechnol. 2013;31:213-9. 
12. Abo RP, Ducar M, Garcia EP, et al. BreaKmer: detection of structural variation in targeted massively parallel sequencing data using kmers. Nucleic Acids Res. 2015;43:e19.

13. Pasqualucci L, Neumeister P, Goossens T, et al. Hypermutation of multiple proto-oncogenes in B-cell diffuse large-cell lymphomas. Nature. 2001;412:341-6.

14. Yao Z, Yaeger R, Rodrik-Outmezguine VS, et al. Tumours with class 3 BRAF mutants are sensitive to the inhibition of activated RAS. Nature. 2017;548:234-8.

15. Khodabakhshi AH, Morin RD, Fejes AP, et al. Recurrent targets of aberrant somatic hypermutation in lymphoma. Oncotarget. 2012;3:1308-19.

16. Liu Q, Tomaszewicz K, Hutchinson L, et al. Somatic mutations in histiocytic sarcoma identified by next generation sequencing. Virchows Arch. 2016;469:233-41.

17. Facchetti F, Pileri SA, Lorenzi L, et al. Histiocytic and dendritic cell neoplasms: what have we learnt by studying 67 cases. Virchows Arch. 2017;471:467-89.

18. Kordes M, Röring M, Heining $\mathrm{C}$, et al. Cooperation of BRAFF595L and mutant HRAS in histiocytic sarcoma provides new insights into oncogenic BRAF signaling. Leukemia. 2016;30:937-46.

19. Voruz S, Cairoli A, Naveiras O, et al. Response to MEK inhibition with trametinib and tyrosine kinase inhibition with imatinib in multifocal histiocytic sarcoma. Haematologica. 2018;103: e39-41.

20. Gounder MM, Solit DB, Tap WD. Trametinib in histiocytic sarcoma with an activating MAP2K1 (MEK1) mutation. N Engl J Med. 2018;378:1945-7.

21. Wagle N, Emery C, Berger MF, et al. Dissecting therapeutic resistance to RAF inhibition in melanoma by tumor genomic profiling. J Clin Oncol. 2011;29:3085-96.

22. Waterfall JJ, Arons E, Walker RL, et al. High prevalence of MAP2K1 mutations in variant and IGHV4-34-expressing hairycell leukemias. Nat Genet. 2014;46:8-10.

23. Kerr EM, Gaude E, Turrell FK, et al. Mutant Kras copy number defines metabolic reprogramming and therapeutic susceptibilities. Nature. 2016;531:110-3.

24. Tartaglia M, Niemeyer CM, Fragale A, et al. Somatic mutations in PTPN11 in juvenile myelomonocytic leukemia, myelodysplastic syndromes and acute myeloid leukemia. Nat Genet. 2003;34:148-50.

25. Ardini E, Bosotti R, Borgia AL, et al. The TPM3-NTRK1 rearrangement is a recurring event in colorectal carcinoma and is associated with tumor sensitivity to TRKA kinase inhibition. Mol Oncol. 2014;8:1459-507.

26. Drilon A, Laetsch TW, Kummar S, et al. Efficacy of larotrectinib in $\mathrm{T} R K$ fusion-positive cancers in adults and children. $\mathrm{N}$ Engl $\mathrm{J}$ Med. 2018;378:731-9.

27. Idbaih A, Mokhtari K, Emile J-F, et al. Dramatic response of a BRAF V600E-mutated primary CNS histiocytic sarcoma to vemurafenib. Neurology. 2014;83:1478-80.

28. Javadi M, Richmond TD, Huang K, et al. CBL linker region and RING finger mutations lead to enhanced granulocyte-macrophage colony-stimulating factor (GM-CSF) signaling via elevated levels of JAK2 and LYN. J Biol Chem. 2013;288:19459-70.

29. Rathinam C, Thien CBF, Flavell RA, et al. Myeloid leukemia development in c-Cbl RING finger mutant mice is dependent on FLT3 signaling. Cancer Cell. 2010;18:341-52.

30. Bazhenova L, Neuteboom S, Chen I, et al. P3.01-048 CBL mutations as potential mediators of EGFR TKI resistance effectively treated with sitravatinib. J Thorac Oncol. 2017;12:S2220.

31. Denning G, Jean-Joseph B, Prince C, et al. A short N-terminal sequence of PTEN controls cytoplasmic localization and is required for suppression of cell growth. Oncogene. 2007;26:3930-40.

32. Lucas CL, Zhang Y, Venida A, et al. Heterozygous splice mutation in PIK3R1 causes human immunodeficiency with lymphoproliferation due to dominant activation of PI3K. J Exp Med. 2014;211:2537-47.

33. Sharpless NE. INK4a/ARF: a multifunctional tumor suppressor locus. Mutat Res. 2005;576:22-38.

34. Kumar R, Khan SP, Joshi D-D, et al. Pediatric histiocytic sarcoma clonally related to precursor B-cell acute lymphoblastic leukemia with homozygous deletion of CDKN2A encoding p16INK4A. Pediatr Blood Cancer. 2011;56:307-10.

35. Carrasco DR, Fenton T, Sukhdeo K, et al. The PTEN and INK4A/ ARF tumor suppressors maintain myelolymphoid homeostasis and cooperate to constrain histiocytic sarcoma development in humans. Cancer Cell. 2006;9:379-90.

36. Gatalica Z, Bilalovic N, Palazzo JP, et al. Disseminated histiocytoses biomarkers beyond BRAFV600E: frequent expression of PD-L1. Oncotarget. 2015;6:19819-25.

37. Xu J, Sun HH, Fletcher CDM, et al. Expression of programmed cell death 1 ligands (PD-L1 and PD-L2) in histiocytic and dendritic cell disorders. Am J Surg Pathol. 2016;40:443-53.

38. Roemer MGM, Advani RH, Ligon AH, et al. $P D-L 1$ and $P D-L 2$ genetic alterations define classical hodgkin lymphoma and predict outcome. J Clin Oncol. 2016;34:2690-7.

39. Zeng K, Wang Z, Ohshima K, et al. BRAF V600E mutation correlates with suppressive tumor immune microenvironment and reduced disease-free survival in Langerhans cell histiocytosis. Oncoimmunology. 2016;5:e1185582.

40. Rossi D, Cerri M, Capello D, et al. Aberrant somatic hypermutation in primary mediastinal large B-cell lymphoma [16]. Leukemia. 2005;19:2363-6.

41. Ansari J, Naqash AR, Munker R, et al. Histiocytic sarcoma as a secondary malignancy: pathobiology, diagnosis, and treatment. Eur J Haematol. 2016;97:9-16.

42. Chen W, Lau SK, Fong D, et al. High frequency of clonal immunoglobulin receptor gene rearrangements in sporadic histiocytic/dendritic cell sarcomas. Am J Surg Pathol. 2009;33:863-73.

43. Gao J, Aksoy BA, Dogrusoz U, et al. Integrative analysis of complex cancer genomics and clinical profiles using the cBioPortal. Sci Signal. 2013;6:pl1.

44. Cerami E, Gao J, Dogrusoz U, et al. The cBio cancer genomics portal: an open platform for exploring multidimensional cancer genomics data. Cancer Discov. 2012;2:401-4. 